\title{
Abstract Poisson summation formulas over homogeneous spaces of compact groups
}

\author{
Arash Ghaani Farashahi ${ }^{1}$
}

Received: 12 June 2016 / Revised: 3 November 2016 / Accepted: 9 November 2016 /

Published online: 18 November 2016

(C) The Author(s) 2016. This article is published with open access at Springerlink.com

\begin{abstract}
This paper presents the abstract notion of Poisson summation formulas for homogeneous spaces of compact groups. Let $G$ be a compact group, $H$ be a closed subgroup of $G$, and $\mu$ be the normalized $G$-invariant measure over the left coset space $G / H$ associated to the Weil's formula. We prove that the abstract Fourier transform over $G / H$ satisfies a generalized version of the Poisson summation formula.
\end{abstract}

Keywords Compact group $\cdot$ Homogeneous space $\cdot$ Dual homogeneous space $\cdot$ Fourier transform $\cdot$ Plancherel (trace) formula $\cdot$ Inversion formula $\cdot$ Poisson summation formula

Mathematics Subject Classification Primary 20G05 - 43A85; Secondary 43A30 . 43A90

\section{Introduction}

The classical Poisson summation formula is a spectacular result with many important applications in physics and engineering [1-3]. Various connections of the Poisson formula with group representations have been discovered for a some time already. In the most natural domain of commutative groups this can be already found in $[4,14]$. Inspiring connections with the Heisenberg group have been discussed in [1].

The abstract aspect of harmonic analysis over homogeneous spaces of compact non-Abelian groups, more precisely, left (resp. right) coset spaces of non-normal sub-

Arash Ghaani Farashahi

arash.ghaani.farashahi@univie.ac.at; ghaanifarashahi@hotmail.com

1 Numerical Harmonic Analysis Group (NuHAG), Faculty of Mathematics, University of Vienna, Vienna, Austria 
groups of compact non-Abelian groups is placed as building blocks for coherent states analysis, constructive approximation, and particle physics, see [4,10,11,15-19] and references therein. Over the last decades, abstract and computational aspects of Fourier transforms and Plancherel formulas over symmetric spaces have achieved significant popularity in geometric analysis, mathematical physics, and scientific computing, see $[6,8,9]$ and references therein.

Let $G$ be a compact group and $H$ be a closed subgroup of $G$. Then the left coset space $G / H$ is a compact homogeneous space, where $G$ acts on it via the left action. Let $\mu$ be the normalized $G$-invariant measure on the compact homogeneous space $G / H$ associated to the Weil's formula with respect to the probability measures of $G$ and $H$. This article consists of theoretical aspects of a unified approach to the nature of abstract Poisson summation formulas over homogeneous spaces of compact groups.

This paper is organized as follows. Section 2 is devoted to fixing notation and preliminaries including a summary on non-Abelian Fourier analysis over compact groups and classical results on abstract harmonic analysis over compact homogeneous spaces. In Sect. 3, we present some abstract aspects of harmonic analysis on the Banach functions spaces over the compact homogeneous space $G / H$. We then introduce the abstract notion of dual space $\widehat{G / H}$ for the homogeneous space $G / H$. Next we present the theoretical definition of the abstract operator-valued Fourier transform over the compact homogeneous space $G / H$. The paper is concluded by presenting an abstract generalized version of the Poisson summation formula.

\section{Preliminaries and notation}

Let $\mathcal{H}$ be a separable Hilbert space. An operator $T \in \mathcal{B}(\mathcal{H})$ is called a HilbertSchmidt operator if for one, hence for any orthonormal basis $\left\{e_{k}\right\}$ of $\mathcal{H}$ we have $\sum_{k}\left\|T e_{k}\right\|^{2}<\infty$. The set of all Hilbert-Schmidt operators on $\mathcal{H}$ is denoted by $\operatorname{HS}(\mathcal{H})$ and for $T \in \operatorname{HS}(\mathcal{H})$ the Hilbert-Schmidt norm of $T$ is $\|T\|_{\mathrm{HS}}^{2}=\sum_{k}\left\|T e_{k}\right\|^{2}$. The set $\operatorname{HS}(\mathcal{H})$ is a self adjoint two sided ideal in $\mathcal{B}(\mathcal{H})$ and if $\mathcal{H}$ is finite-dimensional we have $\operatorname{HS}(\mathcal{H})=\mathcal{B}(\mathcal{H})$. An operator $T \in \mathcal{B}(\mathcal{H})$ is trace-class, whenever $\|T\|_{\operatorname{tr}}=\operatorname{tr}[|T|]<$ $\infty$, if $\operatorname{tr}[T]=\sum_{k}\left\langle T e_{k}, e_{k}\right\rangle$ and $|T|=\left(T T^{*}\right)^{1 / 2}$ [20].

Let $G$ be a compact group with the probability Haar measure $d x$. Then each irreducible representation of $G$ is finite dimensional and every unitary representation of $G$ is a direct sum of irreducible representations, see $[4,10]$. The set of of all unitary equivalence classes of irreducible unitary representations of $G$ is denoted by $\widehat{G}$. This definition of $\widehat{G}$ is in essential agreement with the classical definition when $G$ is Abelian, since each character of an Abelian group is a one dimensional representation of $G$. If $\pi$ is any unitary representation of $G$, for $\zeta, \xi \in \mathcal{H}_{\pi}$ the functions $\pi_{\zeta, \xi}(x)=\langle\pi(x) \zeta, \xi\rangle$ are called matrix elements of $\pi$. If $\left\{e_{j}\right\}$ is an orthonormal basis for $\mathcal{H}_{\pi}$, then $\pi_{i j}$ means $\pi_{e_{i}, e_{j}}$. The notation $\mathcal{E}_{\pi}$ is used for the linear span of the matrix elements of $\pi$ and the notation $\mathcal{E}$ is used for the linear span of $\bigcup_{[\pi] \in \widehat{G}} \mathcal{E}_{\pi}$. Then Peter-Weyl Theorem $[4,10]$ guarantees that if $G$ is a compact group, $\mathcal{E}$ is uniformly dense in $\mathcal{C}(G), L^{2}(G)=\bigoplus_{[\pi] \in \widehat{G}} \mathcal{E}_{\pi}$, and $\left\{d_{\pi}^{-1 / 2} \pi_{i j}: i, j=1 \ldots d_{\pi},[\pi] \in \widehat{G}\right\}$ is an orthonormal basis for $L^{2}(G)$. For $f \in L^{1}(G)$ and $[\pi] \in \widehat{G}$, the Fourier transform of $f$ at $\pi$ is defined in the weak sense as an operator in $\mathcal{B}\left(\mathcal{H}_{\pi}\right)$ by 


$$
\widehat{f}(\pi)=\int_{G} f(x) \pi(x)^{*} d x
$$

If $\pi(x)$ is represented by the matrix $\left(\pi_{i j}(x)\right) \in \mathbb{C}^{d_{\pi} \times d_{\pi}}$. Then $\widehat{f}(\pi) \in \mathbb{C}^{d_{\pi}} \times d_{\pi}$ is the matrix with entries given by $\widehat{f}(\pi)_{i j}=d_{\pi}^{-1} c_{j i}^{\pi}(f)$ which satisfies

$$
\sum_{i, j=1}^{d_{\pi}} c_{i j}^{\pi}(f) \pi_{i j}(x)=d_{\pi} \sum_{i, j=1}^{d_{\pi}} \widehat{f}(\pi)_{j i} \pi_{i j}(x)=d_{\pi} \operatorname{tr}[\widehat{f}(\pi) \pi(x)],
$$

where $c_{i, j}^{\pi}(f)=d_{\pi}\left\langle f, \pi_{i j}\right\rangle_{L^{2}(G)}$. Then as a consequence of Peter-Weyl Theorem we get

$$
\|f\|_{L^{2}(G)}^{2}=\sum_{[\pi] \in \widehat{G}} d_{\pi}\|\widehat{f}(\pi)\|_{\mathrm{HS}}^{2}
$$

and

$$
f(x)=\sum_{[\pi] \in \widehat{G}} d_{\pi} \operatorname{tr}[\widehat{f}(\pi) \pi(x)] \text { for a.e. } x \in G .
$$

Let $H$ be a closed subgroup of $G$ with the probability Haar measure $d h$. The left coset space $G / H$ is considered as a compact homogeneous space that $G$ acts on it from the left and $q: G \rightarrow G / H$ given by $x \mapsto q(x):=x H$ is the surjective canonical map. The classical aspects of abstract harmonic analysis on locally compact homogeneous spaces are quite well studied by several authors, see $[10,11,21]$ and references therein. If $G$ is compact, each transitive $G$-space can be considered as a left coset space $G / H$ for some closed subgroup $H$ of $G$. The function space $\mathcal{C}(G / H)$ consists of all functions $T_{H}(f)$, where $f \in \mathcal{C}(G)$ and

$$
T_{H}(f)(x H)=\int_{H} f(x h) d h .
$$

Let $\mu$ be a Radon measure on $G / H$ and $x \in G$. The translation $\mu_{x}$ of $\mu$ is defined by $\mu_{x}(E)=\mu(x E)$, for all Borel subsets $E$ of $G / H$. The measure $\mu$ is called $G$-invariant if $\mu_{x}=\mu$, for all $x \in G$. The homogeneous space $G / H$ has a normalized $G$-invariant measure $\mu$, which satisfies the following Weil's formula [4,21]

$$
\int_{G / H} T_{H}(f)(x H) d \mu(x H)=\int_{G} f(x) d x \text { for all } f \in L^{1}(G),
$$

and also the following norm-decreasing formula

$$
\left\|T_{H}(f)\right\|_{L^{1}(G / H, \mu)} \leq\|f\|_{L^{1}(G)}, \text { for all } f \in L^{1}(G) .
$$




\section{Abstract harmonic analysis over homogeneous spaces of compact groups}

Throughout this paper we assume that $G$ is a compact group with the probability Haar measure $d x, H$ is a closed subgroup of $G$ with the probability Haar measure $d h$, and $\mu$ is the normalized $G$-invariant measure on the compact homogeneous space $G / H$ associated to the Weil's formula (2.5) with respect to the probability measures of $G$ and $H$. From now on, we may say $\mu$ is the normalized $G$-invariant measure over the compact homogeneous space $G / H$, at times.

This section is devoted to present a list of useful results concerning the classical study for abstract harmonic analysis of Banach function spaces over homogeneous spaces of compact groups, for proofs we refer the readers to see $[5,7,8]$.

The following proposition shows that the linear map $T_{H}: \mathcal{C}(G) \rightarrow \mathcal{C}(G / H)$ is uniformly continuous.

Proposition 3.1 Let $H$ be a closed subgroup of a compact group $G$. The linear map $T_{H}:\left(\mathcal{C}(G),\|.\|_{\text {sup }}\right) \rightarrow\left(\mathcal{C}(G / H),\|.\|_{\text {sup }}\right)$ is uniformly continuous.

Next theorem proves that the linear map $T_{H}$ is norm-decreasing in other $L^{p}$-spaces, when $p>1$.

Theorem 3.2 Let $H$ be a closed subgroup of a compact group $G, \mu$ be the normalized $G$-invariant measure on $G / H$, and $p \geq 1$. The linear map $T_{H}: \mathcal{C}(G) \rightarrow \mathcal{C}(G / H)$ has an extension to a bounded linear map from $L^{p}(G)$ onto $L^{p}(G / H, \mu)$.

As an immediate consequence of Theorem 3.2 we deduce the following corollary.

Corollary 3.3 Let $H$ be a closed subgroup of a compact group $G, \mu$ be the normalized $G$-invariant measure on $G / H$, and $p \geq 1$. Let $\varphi \in L^{p}(G / H, \mu)$ and $\varphi_{q}:=\varphi \circ q$. Then $\varphi_{q} \in L^{p}(G)$ with

$$
\left\|\varphi_{q}\right\|_{L^{p}(G)}=\|\varphi\|_{L^{p}(G / H, \mu)} .
$$

Let $\mathcal{J}^{2}(G, H):=\left\{f \in L^{2}(G): T_{H}(f)=0\right\}$ and $\mathcal{J}^{2}(G, H)^{\perp}$ be the orthogonal complement of the closed subspace $\mathcal{J}^{2}(G, H)$ in $L^{2}(G)$.

Proposition 3.4 Let $H$ be a closed subgroup of a compact group $G$ and $\mu$ be the normalized $G$-invariant measure on $G / H$. Then $T_{H}: L^{2}(G) \rightarrow L^{2}(G / H, \mu)$ is a partial isometric linear map.

The following corollaries are straightforward consequences of Proposition 3.4.

Corollary 3.5 Let $H$ be a closed subgroup of a compact group $G$. Let $P_{\mathcal{J}^{2}(G, H)}$ and $P_{\mathcal{J}^{2}(G, H)^{\perp}}$ be the orthogonal projections onto the closed subspaces $\mathcal{J}^{2}(G, H)$ and $\mathcal{J}^{2}(G, H)^{\perp}$ respectively. Then, for $f \in L^{2}(G)$ and a.e. $x \in G$, we have

$$
P_{\mathcal{J}^{2}(G, H)^{\perp}}(f)(x)=T_{H}(f)(x H), \quad P_{\mathcal{J}^{2}(G, H)}(f)(x)=f(x)-T_{H}(f)(x H) .
$$

Corollary 3.6 Let $H$ be a compact subgroup of a compact group $G$ and $\mu$ be the normalized $G$-invariant measure on $G / H$. Then 
(1) $\mathcal{J}^{2}(G, H)^{\perp}=\left\{\psi_{q}=\psi \circ q: \psi \in L^{2}(G / H, \mu)\right\}$.

(2) For $f \in \mathcal{J}^{2}(G, H)^{\perp}$ and $h \in H$, we have $R_{h} f=f$.

(3) For $\psi \in L^{2}(G / H, \mu)$, we have $\left\|\psi_{q}\right\|_{L^{2}(G)}=\|\psi\|_{L^{2}(G / H, \mu)}$.

(4) For $f, g \in \mathcal{J}^{2}(G, H)^{\perp}$, we have $\left\langle T_{H}(f), T_{H}(g)\right\rangle_{L^{2}(G / H, \mu)}=\langle f, g\rangle_{L^{2}(G)}$.

Remark 3.7 Invoking Corollary 3.6, one can regard the Hilbert space $L^{2}(G / H, \mu)$ as a closed subspace of $L^{2}(G)$, that is the closed subspace consists of all $f \in L^{2}(G)$ which satisfies $R_{h} f=f$, for all $h \in H$. Then Theorem 3.2 and Proposition 3.4 guarantee that the linear map

$$
T_{H}: L^{2}(G) \rightarrow L^{2}(G / H, \mu) \subset L^{2}(G)
$$

is an orthogonal projection onto $L^{2}(G / H, \mu)$.

\section{Abstract duality over homogeneous spaces of compact groups}

In this section, we present the abstract notion of dual homogeneous spaces associated to homogeneous spaces of compact groups [8].

For a closed subgroup $H$ of $G$, let

$$
H^{\perp}=\{[\pi] \in \widehat{G}: \pi(h)=I \quad \text { for all } h \in H\} .
$$

Then by definition we have

$$
H^{\perp} \subseteq \widehat{G}
$$

If $G$ is Abelian, each closed subgroup $H$ of $G$ is normal and the compact group $G / H$ is Abelian and so $\widehat{G / H}$ is precisely the set of all characters (one dimensional irreducible representations) of $G$ which are constant on $H$, that is precisely $H^{\perp}$. If $G$ is a nonAbelian group and $H$ is a closed normal subgroup of $G$, then the dual space $\widehat{G / H}$ which is the set of all unitary equivalence classes of unitary representations of the quotient group $G / H$, has meaning and it is well-defined. Indeed, $G / H$ is a non-Abelian group. In this case, the map $\Phi: \widehat{G / H} \rightarrow H^{\perp}$ defined by $\sigma \mapsto \Phi(\sigma):=\sigma \circ q$ is a Borel isomorphism and $\widehat{G / H}=H^{\perp}$, see [4,19]. Thus if $H$ is normal, $H^{\perp}$ coincides with the classic definitions of the dual space either when $G$ is Abelian or non-Abelian.

For a closed subgroup $H$ of $G$ and a continuous unitary representation $\left(\pi, \mathcal{H}_{\pi}\right)$ of $G$, define

$$
T_{H}^{\pi}:=\int_{H} \pi(h) d h
$$

where the operator valued integral (4.3) is considered in the weak sense.

In other words,

$$
\left\langle T_{H}^{\pi} \zeta, \xi\right\rangle=\int_{H}\langle\pi(h) \zeta, \xi\rangle d h, \text { for } \zeta, \xi \in \mathcal{H}_{\pi}
$$


The function $h \mapsto\langle\pi(h) \zeta, \xi\rangle$ is bounded and continuous on $H$. Since $H$ is compact, the right integral is the ordinary integral of a function in $L^{1}(H)$. Therefore, $T_{H}^{\pi}$ is a bounded linear operator on $\mathcal{H}_{\pi}$ with $\left\|T_{H}^{\pi}\right\| \leq 1$.

Proposition 4.1 Let $H$ be a closed subgroup of a compact group $G$ and $\left(\pi, \mathcal{H}_{\pi}\right)$ be a continuous unitary representation of $G$ with $T_{H}^{\pi} \neq 0$. Then the linear operator $T_{H}^{\pi}$ is an orthogonal projection.

Definition 4.2 Let $H$ be a closed subgroup of a compact group $G$. Then we define the dual space of $G / H$, as the subset of $\widehat{G}$ which is given by

$$
\widehat{G / H}:=\left\{[\pi] \in \widehat{G}: T_{H}^{\pi} \neq 0\right\}=\left\{[\pi] \in \widehat{G}: \int_{H} \pi(h) d h \neq 0\right\} .
$$

Evidently, any closed subgroup $H$ of $G$ satisfies

$$
H^{\perp} \subseteq \widehat{G / H}
$$

Let $\left(\pi, \mathcal{H}_{\pi}\right)$ be a continuous unitary representation of $G$ such that $T_{H}^{\pi} \neq 0$. Then the functions $\pi_{\zeta, \xi}^{H}: G / H \rightarrow \mathbb{C}$ defined by

$$
\pi_{\zeta, \xi}^{H}(x H):=\left\langle\pi(x) T_{H}^{\pi} \zeta, \xi\right\rangle \text { for } x H \in G / H,
$$

for $\xi, \zeta \in \mathcal{H}_{\pi}$, are called $H$-matrix elements of $\left(\pi, \mathcal{H}_{\pi}\right)$.

For $x H \in G / H$ and $\zeta, \xi \in \mathcal{H}_{\pi}$, we have

$$
\begin{aligned}
\left|\pi_{\zeta, \xi}^{H}(x H)\right| & =\left|\left\langle\pi(x) T_{H}^{\pi} \zeta, \xi\right\rangle\right| \\
& \leq\left\|\pi(x) T_{H}^{\pi} \zeta\right\|\|\xi\| \leq\left\|T_{H}^{\pi} \zeta\right\|\|\xi\| \leq\|\zeta\|\|\xi\| .
\end{aligned}
$$

Also, we can write

$$
\pi_{\zeta, \xi}^{H}(x H)=\left\langle\pi(x) T_{H}^{\pi} \zeta, \xi\right\rangle=\pi_{T_{H}^{\pi} \zeta, \xi}(x) .
$$

Invoking definition of the linear map $T_{H}$ and $T_{H}^{\pi}$, we have

$$
\begin{aligned}
T_{H}\left(\pi_{\zeta, \xi}\right)(x H) & =\int_{H} \pi_{\zeta, \xi}(x h) d h \\
& =\int_{H}\langle\pi(x h) \zeta, \xi\rangle d h \\
& =\int_{H}\langle\pi(x) \pi(h) \zeta, \xi\rangle d h \\
& =\left\langle\pi(x)\left(\int_{H} \pi(h) d h\right) \zeta, \xi\right\rangle=\left\langle\pi(x) T_{H}^{\pi} \zeta, \xi\right\rangle,
\end{aligned}
$$

which implies

$$
T_{H}\left(\pi_{\zeta, \xi}\right)=\pi_{\zeta, \xi}^{H}
$$


Next we show that the reverse inclusion of (4.6) holds, if $H$ is a normal subgroup of $G$.

Theorem 4.3 Let $H$ be a closed normal subgroup of a compact group $G$. Then

$$
\widehat{G / H}=H^{\perp} \text {. }
$$

Proof Let $H$ be a closed normal subgroup of a compact group $G$. It is sufficient to show that $\widehat{G / H} \subseteq H^{\perp}$. Let $[\pi] \in \widehat{G / H}$ be given. Due to the normality of $H$ in $G$, for all $x \in G$ the map $\tau_{x}: H \rightarrow H$ given by $h \mapsto \tau_{x}(h):=x^{-1} h x$ belongs to $\operatorname{Aut}(H)$ and $x^{-1} H x=H$. Invoking compactness of $G$ we have $d\left(\tau_{x}(h)\right)=d h$, for $x \in G$. Now, for $x \in G$ we get

$$
\begin{aligned}
\int_{H} \pi(h) d h & =\int_{x H x^{-1}} \pi\left(\tau_{x}(h)\right) d\left(\tau_{x}(h)\right) \\
& =\int_{H} \pi\left(\tau_{x}(h)\right) d h \\
& =\int_{H} \pi(x)^{*} \pi(h) \pi(x) d h \\
& =\pi(x)^{*}\left(\int_{H} \pi(h) d h\right) \pi(x)=\pi(x)^{*} T_{H}^{\pi} \pi(x) .
\end{aligned}
$$

Therefore, $\pi(x) T_{H}^{\pi}=T_{H}^{\pi} \pi(x)$ for $x \in G$, which implies $T_{H}^{\pi} \in \mathcal{C}(\pi)$. Irreducibility of $\pi$ guarantees that $T_{H}^{\pi}=\alpha I$ for some non-zero $\alpha \in \mathbb{C}$ with $|\alpha| \leq 1$. Thus, for $t \in H$, we can write

$$
\begin{aligned}
\pi(t) & =\alpha^{-1} \pi(t) \alpha I \\
& =\alpha^{-1} \pi(t) T_{H}^{\pi} \\
& =\alpha^{-1} \int_{H} \pi(t h) d h \\
& =\alpha^{-1} \int_{H} \pi(h) d h \\
& =\alpha^{-1} T_{H}^{\pi}=I,
\end{aligned}
$$

which implies $[\pi] \in H^{\perp}$.

\section{Abstract Poisson summation formulas over homogeneous spaces of compact groups}

Throughout this section, we present the abstract notion of operator-valued Fourier transforms and Poisson summation formulas over homogeneous spaces of compact groups. It is still assumed that $H$ is a closed subgroup of a compact group $G$ and $\mu$ is the normalized $G$-invariant measure on the compact homogeneous space $G / H$. 
Let $\varphi \in L^{1}(G / H, \mu)$ and $[\pi] \in \widehat{G / H}$. The Fourier transform of $\varphi$ at $[\pi]$ is defined as the operator

$$
\mathcal{F}_{G / H}(\varphi)(\pi)=\widehat{\varphi}(\pi):=\int_{G / H} \varphi(x H) \Gamma_{\pi}(x H)^{*} d \mu(x H),
$$

on the Hilbert space $\mathcal{H}_{\pi}$, where for $x H \in G / H$ the notation $\Gamma_{\pi}(x H)$ stands for the bounded linear operator on $\mathcal{H}_{\pi}$ satisfying

$$
\left\langle\zeta, \Gamma_{\pi}(x H) \xi\right\rangle=\left\langle\zeta, \pi(x) T_{H}^{\pi} \xi\right\rangle
$$

for all $\zeta, \xi \in \mathcal{H}_{\pi}$

Remark 5.1 Let $H$ be a closed normal subgroup of a compact group $G$ and $\mu$ be the normalized $G$-invariant measure over the left coset space $G / H$ associated to the Weil's formula. Then it is easy to check that $\mu$ is a Haar measure of the compact quotient group $G / H$ and by Theorem 4.3 we have $\widehat{G / H}=H^{\perp}$. Also, for each $\varphi \in L^{1}(G / H, \mu)$ and $[\pi] \in H^{\perp}$, we have

$$
\widehat{\varphi}(\pi)=\int_{G / H} \varphi(x H) \pi(x)^{*} d \mu(x H) .
$$

Thus, we deduce that the abstract Fourier transform defined by (5.1) coincides with the classical Fourier transform over the compact quotient group $G / H$ if $H$ is normal in $G$.

The operator-valued integral (5.1) is also considered in the weak sense. That is

$$
\langle\widehat{\varphi}(\pi) \zeta, \xi\rangle=\int_{G / H} \varphi(x H)\left\langle\Gamma_{\pi}(x H)^{*} \zeta, \xi\right\rangle d \mu(x H),
$$

for all $\zeta, \xi \in \mathcal{H}_{\pi}$.

In other words, for $[\pi] \in \widehat{G / H}$ and $\zeta, \xi \in \mathcal{H}_{\pi}$, we have

$$
\langle\widehat{\varphi}(\pi) \zeta, \xi\rangle=\int_{G / H} \varphi(x H)\left\langle\zeta, \pi(x) T_{H}^{\pi} \xi\right\rangle d \mu(x H) .
$$

Indeed, we can write

$$
\begin{aligned}
\langle\widehat{\varphi}(\pi) \zeta, \xi\rangle & =\int_{G / H} \varphi(x H)\left\langle\Gamma_{\pi}(x H)^{*} \zeta, \xi\right\rangle d \mu(x H) \\
& =\int_{G / H} \varphi(x H)\left\langle\zeta, \Gamma_{\pi}(x H) \xi\right\rangle d \mu(x H) \\
& =\int_{G / H} \varphi(x H)\left\langle\zeta, \pi(x) T_{H}^{\pi} \xi\right\rangle d \mu(x H) .
\end{aligned}
$$


Thus, for $\zeta, \xi \in \mathcal{H}_{\pi}$, we get

$$
\begin{aligned}
|\langle\widehat{\varphi}(\pi) \zeta, \xi\rangle| & =\left|\int_{G / H} \varphi(x H)\left\langle\zeta, \pi(x) T_{H}^{\pi} \xi\right\rangle d \mu(x H)\right| \\
& \leq \int_{G / H}|\varphi(x H)|\left|\left\langle\zeta, \pi(x) T_{H}^{\pi} \xi\right\rangle\right| d \mu(x H) \\
& \leq \int_{G / H}|\varphi(x H)|\|\zeta\|\left\|\pi(x) T_{H}^{\pi} \xi\right\| d \mu(x H) \\
& =\int_{G / H}|\varphi(x H)|\|\zeta\|\left\|T_{H}^{\pi} \xi\right\| d \mu(x H) \\
& \leq \int_{G / H}|\varphi(x H)|\|\zeta\|\|\xi\| d \mu(x H)=\|\zeta\|\|\xi\|\|\varphi\|_{L^{1}(G / H, \mu)}
\end{aligned}
$$

Therefore, $\widehat{\varphi}(\pi)$ is a bounded linear operator on $\mathcal{H}_{\pi}$ satisfying

$$
\|\widehat{\varphi}(\pi)\| \leq\|\varphi\|_{L^{1}(G / H, \mu)} .
$$

From now on, we may use $\widehat{\varphi}(\pi)$ or $\mathcal{F}_{G / H}(\varphi)(\pi)$ at times.

The following proposition gives us the connection of the Fourier transform over the homogeneous space $G / H$ with the Fourier transform on the group $G$.

Proposition 5.2 Let $H$ be a closed subgroup of a compact group $G$ and $\mu$ be the normalized $G$-invariant measure on $G / H$. Let $\varphi \in L^{1}(G / H, \mu)$ and $[\pi] \in \widehat{G / H}$. Then

$$
\mathcal{F}_{G / H}(\varphi)(\pi)=\mathcal{F}_{G}\left(\varphi_{q}\right)(\pi) .
$$

Proof Using (5.4) and also the Weil's formula, for $\zeta, \xi \in \mathcal{H}_{\pi}$, we can write

$$
\begin{aligned}
\langle\widehat{\varphi}(\pi) \zeta, \xi\rangle & =\int_{G / H} \varphi(x H)\left\langle\zeta, \pi(x) T_{H}^{\pi} \xi\right\rangle d \mu(x H) \\
& =\int_{G / H} \varphi(x H) \overline{T_{H}\left(\pi_{\xi, \zeta}\right)(x H)} d \mu(x H) \\
& =\int_{G / H} \varphi(x H) T_{H}\left(\overline{\pi_{\xi, \zeta}}\right)(x H) d \mu(x H) \\
& =\int_{G / H} T_{H}\left(\varphi_{q} \cdot \overline{\pi_{\xi, \zeta}}\right)(x H) d \mu(x H) \\
& =\int_{G} \varphi_{q}(x) \overline{\pi_{\xi, \zeta}(x)} d x \\
& =\int_{G} \varphi_{q}(x)\langle\zeta, \pi(x) \xi\rangle d x \\
& =\int_{G} \varphi_{q}(x)\left\langle\pi(x)^{*} \zeta, \xi\right\rangle d x=\left\langle\widehat{\varphi_{q}}(\pi) \zeta, \xi\right\rangle,
\end{aligned}
$$

which implies (5.6). 
The following proposition states generic properties of the Fourier transform (5.1).

Proposition 5.3 Let $H$ be a closed subgroup of a compact group $G$ and $\mu$ be the normalized $G$-invariant measure on $G / H$. Then

(1) For $f \in L^{1}(G)$ and $[\pi] \in \widehat{G / H}$ we have $\widehat{T_{H}(f)}(\pi)=T_{H}^{\pi} \widehat{f}(\pi)$.

(2) For $\varphi \in L^{1}(G / H, \mu)$ and $[\pi] \in \widehat{G / H}$ we have $T_{H}^{\pi} \widehat{\varphi}(\pi)=\widehat{\varphi}(\pi)$.

(3) For $\varphi \in L^{1}(G / H, \mu)$ and $[\pi] \in \widehat{G / H}$ we have $\operatorname{tr}\left[\widehat{\varphi}(\pi) T_{H}^{\pi}\right]=\operatorname{tr}[\widehat{\varphi}(\pi)]$.

Proof (1) Let $[\pi] \in \widehat{G / H}$ and $\zeta, \xi \in \mathcal{H}_{\pi}$. Invoking definition of the Fourier transform, and using the Weil's formula in the weak sense, we can write

$$
\begin{aligned}
\left\langle\widehat{T_{H}(f)}(\pi) \zeta, \xi\right\rangle & =\int_{G / H} T_{H}(f)(x H)\left\langle\Gamma_{\pi}(x H)^{*} \zeta, \xi\right\rangle d \mu(x H) \\
& =\int_{G / H} T_{H}(f)(x H)\left\langle\zeta, \Gamma_{\pi}(x H) \xi\right\rangle d \mu(x H) \\
& =\int_{G / H} T_{H}(f)(x H)\left\langle\zeta, \pi(x) T_{H}^{\pi} \xi\right\rangle d \mu(x H) \\
& =\int_{G / H} T_{H}\left(f . g_{\zeta, \xi}\right)(x H) d \mu(x H) \\
& =\int_{G} f(x)\left\langle\zeta, \pi(x) T_{H}^{\pi} \xi\right\rangle d x \\
& =\int_{G} f(x)\left\langle\pi(x)^{*} \zeta, T_{H}^{\pi} \xi\right\rangle d x \\
& =\int_{G} f(x)\left\langle T_{H}^{\pi} \pi(x)^{*} \zeta, \xi\right\rangle d x=\left\langle T_{H}^{\pi} \widehat{f}(\pi) \zeta, \xi\right\rangle
\end{aligned}
$$

where $g_{\zeta, \xi}: G \rightarrow \mathbb{C}$ is given by $g_{\zeta, \xi}(x):=\left\langle\zeta, \pi(x) T_{H}^{\pi} \xi\right\rangle$ for $x \in G$.

(2) Let $\varphi \in L^{1}(G / H, \mu)$ and $[\pi] \in \widehat{G / H}$. Then, $\varphi_{q} \in L^{1}(G)$ and $T_{H}\left(\varphi_{q}\right)=\varphi$. Using (1) and since $T_{H}^{\pi}$ is an orthogonal projection, we get

$$
\begin{aligned}
T_{H}^{\pi} \widehat{\varphi}(\pi) & \left.=T_{H}^{\pi} \widehat{T_{H}\left(\varphi_{q}\right.}\right)(\pi) \\
& =T_{H}^{\pi} T_{H}^{\pi} \widehat{\varphi_{q}}(\pi) \\
& =T_{H}^{\pi} \widehat{\varphi_{q}}(\pi) \\
& \left.=\widehat{T_{H}\left(\varphi_{q}\right.}\right)(\pi)=\widehat{\varphi}(\pi) .
\end{aligned}
$$

(3) Let $\varphi \in L^{1}(G / H, \mu)$ and $[\pi] \in \widehat{G / H}$. Then, using (2), we have

$$
\operatorname{tr}\left[\widehat{\varphi}(\pi) T_{H}^{\pi}\right]=\operatorname{tr}\left[T_{H}^{\pi} \widehat{\varphi}(\pi)\right]=\operatorname{tr}[\widehat{\varphi}(\pi)] .
$$

Since $L^{2}(G / H, \mu) \subset L^{1}(G / H, \mu)$, the Fourier transform defined in (5.1) is welldefined for $L^{2}$-functions over the homogeneous space $G / H$.

In the next result, we show that the Fourier transform defined in (5.1) satisfies a generalized version of the Plancherel formula in $L^{2}$-sense. 
Theorem 5.4 Let $H$ be a closed subgroup of a compact group $G$ and $\mu$ be the normalized $G$-invariant measure on $G / H$. Each $\varphi \in L^{2}(G / H, \mu)$ satisfies the Plancherel formula

$$
\sum_{[\pi] \in \widehat{G / H}} d_{\pi}\|\widehat{\varphi}(\pi)\|_{2}^{2}=\|\varphi\|_{L^{2}(G / H, \mu)}^{2} .
$$

Proof See Theorem 4.6 of [8]

The following corollary is a consequence of Theorem 5.4.

Corollary 5.5 Let $H$ be a closed subgroup of a compact group $G$ and $\mu$ be the normalized $G$-invariant measure on $G / H$. Then

(1) For $\varphi, \psi \in L^{2}(G / H, \mu)$, we have

$$
\sum_{[\pi] \in \widehat{G / H}} d_{\pi} \operatorname{tr}\left[\widehat{\varphi}(\pi) \widehat{\psi}(\pi)^{*}\right]=\langle\varphi, \psi\rangle_{L^{2}(G / H, \mu)} .
$$

(2) For $f, g \in \mathcal{J}^{2}(G, H)^{\perp}$, we have

$$
\sum_{[\pi] \in \widehat{G / H}} d_{\pi} \operatorname{tr}\left[\widehat{f}(\pi) \widehat{g}(\pi)^{*}\right]=\langle f, g\rangle_{L^{2}(G)} .
$$

Proof (1) Using the polarization identity and Theorem 5.4, we get (5.8).

(2) Applying the Weil's formula, (5.8), and Corollary 3.6, we get (5.9).

Remark 5.6 Let $H$ be a closed normal subgroup of a compact group $G$ and $\mu$ be the normalized $G$-invariant measure over the left coset space $G / H$ associated to the Weil's formula. Then Theorem 4.3 implies that $\widehat{G / H}=H^{\perp}$ and hence the Plancherel (trace) formula (5.7) reads as follows;

$$
\sum_{[\pi] \in H^{\perp}} d_{\pi}\|\widehat{\varphi}(\pi)\|_{\mathrm{HS}}^{2}=\|\varphi\|_{L^{2}(G / H, \mu)}^{2},
$$

for all $\varphi \in L^{2}(G / H, \mu)$, where

$$
\widehat{\varphi}(\pi)=\int_{G / H} \varphi(x H) \pi(x)^{*} d \mu(x H),
$$

for all $[\pi] \in H^{\perp}$, see Remark 5.1.

Next theorem presents an explicit $L^{2}$-inversion formula for the Fourier transform given in (5.1).

Theorem 5.7 Let $H$ be a closed subgroup of a compact group $G, \mu$ be the normalized $G$-invariant measure on $G / H$, and $\varphi \in L^{2}(G / H, \mu)$. Then the function $\breve{\varphi}: G / H \rightarrow$ $\mathbb{C}$ defined by

$$
x H \mapsto \breve{\varphi}(x H):=\sum_{[\pi] \in \widehat{G / H}} d_{\pi} \operatorname{tr}[\widehat{\varphi}(\pi) \pi(x)],
$$


belongs to $L^{2}(G / H, \mu)$, and we have

$$
\varphi(x H)=\sum_{[\pi] \in \widehat{G / H}} d_{\pi} \operatorname{tr}[\widehat{\varphi}(\pi) \pi(x)] \text { for } \mu-\text { a.e. } x H \in G / H .
$$

Proof Let $\varphi \in L^{2}(G / H)$ and $x \in G$. Then, $\varphi_{q} \in L^{2}(G)$ and hence the series

$$
\sum_{[\pi] \in \widehat{G}} d_{\pi} \operatorname{tr}\left[\widehat{\varphi_{q}}(\pi) \pi(x)\right]
$$

converges and also $y \mapsto \sum_{[\pi] \in \widehat{G}} d_{\pi} \operatorname{tr}\left[\widehat{\varphi_{q}}(\pi) \pi(y)\right]$ defines a function in $L^{2}(G)$. Thus, we deduce that the series

$$
\sum_{[\pi] \in \widehat{G / H}} d_{\pi} \operatorname{tr}[\widehat{\varphi}(\pi) \pi(x)]
$$

is converges, because

$$
\sum_{[\pi] \in \widehat{G / H}} d_{\pi} \operatorname{tr}[\widehat{\varphi}(\pi) \pi(x)]=\sum_{[\pi] \in \widehat{G / H}} d_{\pi} \operatorname{tr}\left[\widehat{\varphi_{q}}(\pi) \pi(x)\right]=\sum_{[\pi] \in \widehat{G}} d_{\pi} \operatorname{tr}\left[\widehat{\varphi_{q}}(\pi) \pi(x)\right] .
$$

Now let $h \in H$. Using Proposition 5.3, we can write

$$
\begin{aligned}
\operatorname{tr}[\widehat{\varphi}(\pi) \pi(x h)] & =\operatorname{tr}\left[T_{H}^{\pi} \widehat{\varphi}(\pi) \pi(x h)\right] \\
& =\operatorname{tr}\left[\widehat{\varphi}(\pi) \pi(x) \pi(h) T_{H}^{\pi}\right] \\
& =\operatorname{tr}\left[\widehat{\varphi}(\pi) \pi(x) T_{H}^{\pi}\right] \\
& =\operatorname{tr}\left[T_{H}^{\pi} \widehat{\varphi}(\pi) \pi(x)\right]=\operatorname{tr}[\widehat{\varphi}(\pi) \pi(x)],
\end{aligned}
$$

which guarantees that $y H \mapsto \sum_{[\pi] \in \widehat{G / H}} d_{\pi} \operatorname{tr}[\widehat{\varphi}(\pi) \pi(y)]$, defines a well-defined function on the left coset space $G / H$. Using (2.3), for $\varphi_{q} \in L^{2}(G)$, we get

$$
\varphi_{q}(x)=\sum_{[\pi] \in \widehat{G}} d_{\pi} \operatorname{tr}\left[\widehat{\varphi_{q}}(\pi) \pi(x)\right], \quad \text { for a.e. } x \in G
$$

Then applying the linear map $T_{H}$ to (5.12), implies (5.11). Indeed, for $x \in G$, we can write

$$
\begin{aligned}
\varphi(x H) & =T_{H}\left(\varphi_{q}\right)(x H) \\
& =\int_{H}\left(\sum_{[\pi] \in \widehat{G}} d_{\pi} \operatorname{tr}\left[\widehat{\varphi_{q}}(\pi) \pi(x h)\right]\right) d h \\
& =\sum_{[\pi] \in \widehat{G}} d_{\pi}\left(\int_{H} \operatorname{tr}\left[\widehat{\varphi_{q}}(\pi) \pi(x h)\right] d h\right)
\end{aligned}
$$




$$
\begin{aligned}
& =\sum_{[\pi] \in \widehat{G}} d_{\pi}\left(\int_{H} \operatorname{tr}\left[\widehat{\varphi_{q}}(\pi) \pi(x) \pi(h)\right] d h\right) \\
& =\sum_{[\pi] \in \widehat{G}} d_{\pi}\left(\int_{H} \operatorname{tr}\left[\pi(h) \widehat{\varphi_{q}}(\pi) \pi(x)\right] d h\right) \\
& =\sum_{[\pi] \in \widehat{G}} d_{\pi} \operatorname{tr}\left[T_{H}^{\pi} \widehat{\varphi_{q}}(\pi) \pi(x)\right] \\
& =\sum_{[\pi] \in \widehat{G / H}} d_{\pi} \operatorname{tr}\left[T_{H}^{\pi} \widehat{\varphi_{q}}(\pi) \pi(x)\right] \\
& =\sum_{[\pi] \in \widehat{G / H}} d_{\pi} \operatorname{tr}\left[T_{H}^{\pi} \widehat{\varphi}(\pi) \pi(x)\right]=\sum_{[\pi] \in \widehat{G / H}} d_{\pi} \operatorname{tr}[\widehat{\varphi}(\pi) \pi(x)] .
\end{aligned}
$$

Remark 5.8 Let $H$ be a closed normal subgroup of a compact group $G$ and $\mu$ be the normalized $G$-invariant measure over the left coset space $G / H$ associated to the Weil's formula. Then Theorem 4.3 implies that $\widehat{G / H}=H^{\perp}$ and hence the $L^{2}$-inversion formula (5.11) reads as follows;

$$
\varphi(x H)=\sum_{[\pi] \in H^{\perp}} d_{\pi} \operatorname{tr}[\widehat{\varphi}(\pi) \pi(x)]
$$

for all $\varphi \in L^{2}(G / H, \mu)$ and $\mu$ - a.e. $x H \in G / H$, where

$$
\widehat{\varphi}(\pi)=\int_{G / H} \varphi(x H) \pi(x)^{*} d \mu(x H),
$$

for all $[\pi] \in H^{\perp}$.

Using the Weil's formula and Theorem 5.7, we conclude the following corollary.

Corollary 5.9 Let $H$ be a closed subgroup of a compact group $G$ and $\mu$ be the normalized $G$-invariant measure on $G / H$. Then

(1) For $\varphi \in L^{2}(G / H, \mu)$, we have

$$
\int_{G} \varphi(x H) d x=\int_{G / H}\left(\sum_{[\pi] \in \widehat{G / H}} d_{\pi} \operatorname{tr}[\widehat{\varphi}(\pi) \pi(x)]\right) d \mu(x H) .
$$

(2) For $f \in \mathcal{J}^{2}(G, H)^{\perp}$, we have

$$
\int_{G} f(x) d x=\int_{G / H}\left(\sum_{[\pi] \in \widehat{G / H}} d_{\pi} \operatorname{tr}[\widehat{f}(\pi) \pi(x)]\right) d \mu(x H) .
$$


Proof (1) Let $\varphi \in L^{2}(G / H, \mu)$. Then we have $\varphi_{q} \in L^{2}(G)$ with $T_{H}\left(\varphi_{q}\right)=\varphi$. Using the Weil's formula and (5.11), we have

$$
\begin{aligned}
\int_{G} \varphi(x H) d x & =\int_{G} \varphi_{q}(x) d x \\
& =\int_{G / H} T_{H}\left(\varphi_{q}\right)(x H) d \mu(x H) \\
& =\int_{G / H} \varphi(x H) d \mu(x H)=\int_{G / H}\left(\sum_{[\pi] \in \widehat{G / H}} d_{\pi} \operatorname{tr}[\widehat{\varphi}(\pi) \pi(x)]\right) d \mu(x H),
\end{aligned}
$$

which proves (5.13).

(2) It is straightforward by (1).

Remark 5.10 Let $H$ be a closed normal subgroup of a compact group $G$ and $\mu$ be the normalized $G$-invariant measure over the left coset space $G / H$ associated to the Weil's formula. Then Theorem 4.3 implies that $\widehat{G / H}=H^{\perp}$ and hence the formulas (5.13) and (5.14) read as follows;

$$
\int_{G} \varphi(x H) d x=\int_{G / H}\left(\sum_{[\pi] \in H^{\perp}} d_{\pi} \operatorname{tr}[\widehat{\varphi}(\pi) \pi(x)]\right) d \mu(x H),
$$

and

$$
\int_{G} f(x) d x=\int_{G / H}\left(\sum_{[\pi] \in H^{\perp}} d_{\pi} \operatorname{tr}[\widehat{f}(\pi) \pi(x)]\right) d \mu(x H),
$$

for all $\varphi \in L^{2}(G / H, \mu)$ and $f \in \mathcal{J}^{2}(G, H)^{\perp}$, where

$$
\widehat{\varphi}(\pi)=\int_{G / H} \varphi(x H) \pi(x)^{*} d \mu(x H),
$$

and

$$
\widehat{f}(\pi)=\int_{G} f(x) \pi(x)^{*} d x
$$

for all $[\pi] \in H^{\perp}$.

The following result can be considered as an abstract generalization of the Poisson summation formula for homogeneous spaces of compact groups. 
Theorem 5.11 Let $H$ be a closed subgroup of a compact group $G$. Each $f \in \mathcal{C}(G)$ satisfies the following Poisson summation type formula

$$
\int_{H} f(x h) d h=\sum_{[\pi] \in \widehat{G / H}} d_{\pi} \operatorname{tr}\left[\widehat{f}(\pi) \pi(x) T_{H}^{\pi}\right], \quad \text { for } x \in G .
$$

Proof Let $\mu$ be the normalized $G$-invariant measure on $G / H$ and $f \in \mathcal{C}(G)$. Then by (5.11), we have

$$
T_{H}(f)(x H)=\sum_{[\pi] \in \widehat{G / H}} d_{\pi} \operatorname{tr}\left[\widehat{T_{H}(f)}(\pi) \pi(x)\right] \text { for a.e. } x H \in G / H
$$

Let $x \in G$. Using Proposition 5.3 and (5.16), we get

$$
\begin{aligned}
\int_{H} f(x h) d h & =T_{H}(f)(x H) \\
& =\sum_{[\pi] \in \widehat{G / H}} d_{\pi} \operatorname{tr}\left[\widehat{T_{H}(f)}(\pi) \pi(x)\right] \\
& =\sum_{[\pi] \in \widehat{G / H}} d_{\pi} \operatorname{tr}\left[T_{H}^{\pi} \widehat{f}(\pi) \pi(x)\right]=\sum_{[\pi] \in \widehat{G / H}} d_{\pi} \operatorname{tr}\left[\widehat{f}(\pi) \pi(x) T_{H}^{\pi}\right],
\end{aligned}
$$

which proves (5.15).

Remark 5.12 Let $H$ be a closed normal subgroup of a compact group $G$ and $\mu$ be the normalized $G$-invariant measure over the left coset space $G / H$ associated to the Weil's formula. Then Theorem 4.3 implies that $\widehat{G / H}=H^{\perp}$ and hence the Poisson summation formula (5.15) reads as follows [12];

$$
\int_{H} f(x h) d h=\sum_{[\pi] \in H^{\perp}} d_{\pi} \operatorname{tr}[\widehat{f}(\pi) \pi(x)]
$$

for all $f \in \mathcal{C}(G)$ and $x \in G$, where

$$
\widehat{f}(\pi)=\int_{G} f(x) \pi(x)^{*} d x
$$

for all $[\pi] \in H^{\perp}$.

Corollary 5.13 Let $H$ be a closed subgroup of a compact group $G$. Each $f \in \mathcal{C}(G)$ satisfies the following Poisson summation type formula

$$
\int_{H} f(h) d h=\sum_{[\pi] \in \widehat{G / H}} d_{\pi} \operatorname{tr}\left[\widehat{f}(\pi) T_{H}^{\pi}\right] .
$$


Remark 5.14 It should be mentioned that in [13] author presented an extension for Poisson summation formulas over homogeneous spaces of compact groups in a different direction. In this framework, Corollary 5.13 coincides with Proposition 4.6 of [13].

Acknowledgements Open access funding provided by University of Vienna.

Open Access This article is distributed under the terms of the Creative Commons Attribution 4.0 International License (http://creativecommons.org/licenses/by/4.0/), which permits unrestricted use, distribution, and reproduction in any medium, provided you give appropriate credit to the original author(s) and the source, provide a link to the Creative Commons license, and indicate if changes were made.

\section{References}

1. Auslander, L., Meyer, Y.: A generalized Poisson summation formula. Appl. Comput. Harmon. Anal. 3(4), 372-376 (1996)

2. Castaneda, J., Heusdens, R., Hendriks, R.: A generalized Poisson summation formula and its application to fast linear convolution. IEEE Signal Process. Lett. 18(9), 501-504 (2011)

3. Dooley, A.H.: A nonabelian version of the shannon sampling theorem. SIAM J. Math. Anal. 20, 624-633 (1989)

4. Folland, G.B.: A Course in Abstract Harmonic Analysis. CRC Press, Boca Raton (1995)

5. Ghaani Farashahi, A.: Convolution and involution on function spaces of homogeneous spaces. Bull. Malays. Math. Sci. Soc. (2) 36(4), 1109-1122 (2013)

6. Ghaani Farashahi, A.: A unified group theoretical method for the partial Fourier analysis on semi-direct product of locally compact groups. Results Math. 67(1-2), 235-251 (2015)

7. Ghaani Farashahi, A.: Abstract convolution function algebras over homogeneous spaces of compact groups. Ill. J. Math. (2016) (to appear)

8. Ghaani Farashahi, A.: Abstract Plancherel (trace) formulas over homogeneous spaces of compact groups. Can. Math. Bull. (2016). doi:10.4153/CMB-2016-037-6

9. Ghaani Farashahi, A.: Abstract relative Fourier transforms over canonical homogeneous spaces of semi-direct product groups with Abelian normal factor. J. Korean Math. Soc. (2016). doi:10.4134/ JKMS.j150610

10. Hewitt, E., Ross, K.A.: Abstract Harmonic Analysis, vol. 1. Springer, Berlin (1963)

11. Hewitt, E., Ross, K.A.: Abstract Harmonic Analysis, vol. 2. Springer, Berlin (1970)

12. Ikeshoji, K.: On Plancherel measures of quotient groups and the Poisson summation formula. Math. Japon. 35(5), 981-984 (1990)

13. Jüstel, D.: The Zak transform on strongly proper G-spaces and its applications, arXiv preprint arXiv:1605.05168 (2016)

14. Kirillov, A.A., Gvishiani, A.D.: Teoremy i zadachi funktsionalnogo analiza, 2nd edn. Nauka, Moscow (1988)

15. Kisil, V.: Calculus of operators: covariant transform and relative convolutions. Banach J. Math. Anal. 8(2), 156-184 (2014)

16. Kisil, V.: Erlangen program at large: an overview. In: Advances in applied analysis, 1-94, Trends Math., Birkhäuser/Springer Basel AG, Basel (2012)

17. Kisil, V.: Geometry of Möbius transformations. Elliptic, parabolic and hyperbolic actions of $S L_{2}(\mathbb{R})$, Imperial College Press, London (2012)

18. Kisil, V.: Relative convolutions. I. Properties and applications. Adv. Math. 147(1), 35-73 (1999)

19. Lipsman, R.: Non-abelian fourier analysis. Bull. Sci. Math., $2^{e}$ series, 98, 209-233 (1974)

20. Murphy, G.J.: C*-Algebras and Operator Theory. Academic Press, INC, Boston, MA (1990)

21. Reiter, H., Stegeman, J.D.: Classical Harmonic Analysis, 2nd edn. Oxford University Press, New York (2000) 